\title{
False Oxygen Consumption Effect and Factors Causing It
}

\author{
M. V. Miniaev, M. B. Belyakova, N. V. Kostiuk, and D. V. Leshchenko \\ Department of Biochemistry and Research Center, Tver State Medical Academy, 4 Sovetskaya Street, Tver 170100, Russia \\ Correspondence should be addressed to M. B. Belyakova, mayabe@yandex.ru
}

Received 24 February 2011; Revised 28 April 2011; Accepted 29 April 2011

Academic Editor: Sibel A. Ozkan

Copyright (C) 2011 M. V. Miniaev et al. This is an open access article distributed under the Creative Commons Attribution License, which permits unrestricted use, distribution, and reproduction in any medium, provided the original work is properly cited.

\begin{abstract}
False oxygen consumption effect characterized by a decrease of the polarographic sensor readings by the introduction of neutral microadditives into the incubation medium was modeled and tested. These neutral microadditives neither consume oxygen nor cause its consumption by other components of the medium. It is shown that microadditives less than $3 \%$ of the volume of incubation medium can cause statistically significant effect of false oxygen consumption more than $4 \%$ of the initial oxygen content. The effect can reach more than $15 \%$ at higher volumes of additives. The most important properties of additives enhancing the effect are low oxygen content, low temperature, and low concentration of oxygen salting out components.
\end{abstract}

\section{Introduction}

The introduction of reagents in the incubation medium is obligatory operation for the polarographic study of oxygen consumption. Additives such as ADP, substrate, or uncoupler precisely start the process of consumption. Even at constant oxygen content in the reaction mixture, the peculiarities of additives capable to decrease the readings of the measuring equipment are neglected. In the best case, this should lead to a distortion of measurement results, and in worst case would ascertain the consumption of oxygen where it is actually not. Under such circumstances, the result appears to be more significant than expected. Here, this phenomenon would be called "false oxygen consumption effect."

The most common properties of the additives, which can cause false oxygen consumption, include: (1) absence or low content of dissolved oxygen, (2) low temperature, (3) low levels of oxygen salting-out components, and (4) high viscosity.

The introduction of microadditives $(10-500 \mu \mathrm{L})$ that have at least one of the above listed properties should lead to a noticeable reduction in either the concentration or partial pressure of oxygen in medium. Moreover, viscous additives would increase the thickness of the diffusion layer of the measuring electrodes. This would result in the reduction of polarographic oxygen sensor readings, which could be incorrectly interpreted as oxygen consumption by the biological object of study.

The effect of false oxygen consumption accompanies almost every study of respiration in liquid incubation media, whereas in the literature there are separate efforts to identify the effect and to understand its mechanisms [1-5]. So a simple system was used as a model where certain parameters of microadditives were manipulated. This demonstrated the quantitative aspect of false oxygen consumption, achieving the aim of the study.

\section{Methods}

2.1. Measuring Cell. A measuring cell of volume $4 \mathrm{~mL}$ (Figure 1), was equipped with silver-zinc polarographic oxygen sensor, a Clark electrode [6] in the modification of Mancy [7], and thermostabilized at $37^{\circ} \mathrm{C}\left( \pm 0.1^{\circ} \mathrm{C}\right)$. Sensor readings were recorded by an oximeter N5221 (Poland). To reduce the gas exchange between the incubation medium and atmosphere, floating plastic lid was placed on the surface of the medium. This allowed addition of any volume to cell without loss of fluid. However, the lid did not completely stop the diffusion of oxygen from the atmosphere into the incubation medium. Therefore, the raw data were corrected as published previously [8]. 
TABLE 1: Parameters of oxygen-free additives used in the study of the factors causing false oxygen consumption.

\begin{tabular}{lccc}
\hline no. & Investigated factor & Salt concentration & Thickener \\
\hline 1 & Oxygen concentration & $\mathrm{KCl}, 60 \mathrm{~g} / \mathrm{L}$ & - \\
2 & Temperature & $\mathrm{KCl}, 60 \mathrm{~g} / \mathrm{L}$ & - \\
3 & Salting out & $0 \mathrm{~g} / \mathrm{L}\left(\mathrm{H}_{2} \mathrm{O}\right)$ & - \\
4 & Salting out & $\mathrm{KCl}, 120 \mathrm{~g} / \mathrm{L}$ & - \\
5 & Viscosity & $\mathrm{KCl}, 60 \mathrm{~g} / \mathrm{L}$ & 37 \\
\hline
\end{tabular}

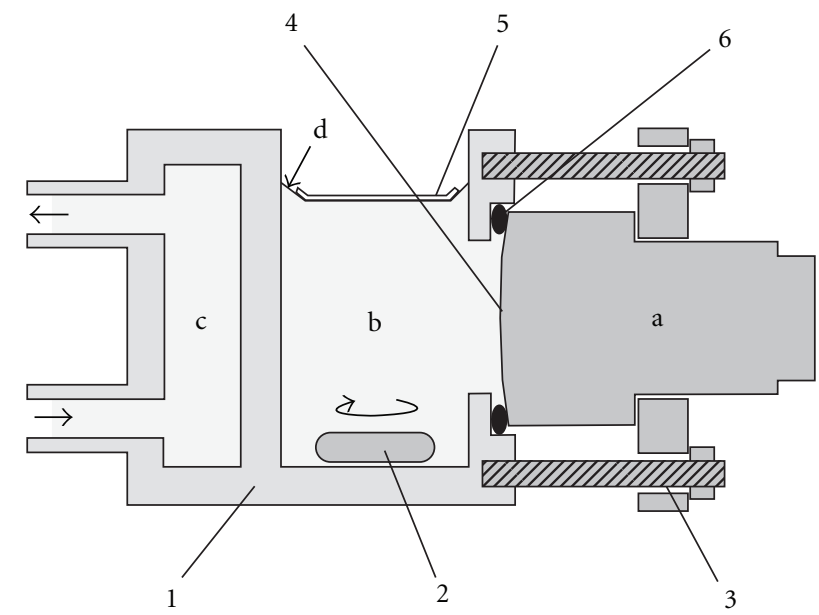

Figure 1: Thermostated measuring cell. (a) oxygen sensor, (b) the incubation medium, (c) water jacket, and (d) a place to introduction of additives. (1) the cell body (PMMA), (2) magnetic stirrer, (3) sensor mount, (4) sensor membrane (Teflon, $20 \mu \mathrm{m}$ ), (5) floating lid (polyethylene), and (6) gasket (rubber).

2.2. Model of the Incubation Medium. To exclude the osmotic effects on the membrane of the oxygen sensor and to facilitate the calculation, instead of the real incubation medium, a simple model was used. This model was a solution of $\mathrm{KCl}$ with a concentration of $60 \mathrm{~g} / \mathrm{L}$, which had the same composition and osmotic pressure as the sensor electrolyte. It allowed to calculate the concentration of oxygen basing on reference data. This solution was saturated with oxygen by continuous flow of atmospheric air enriched with water vapor in a thermostated flask at $37^{\circ} \mathrm{C}$. The concentration of $\mathrm{O}_{2}$ in the medium was $1.54 \times 10^{-7} \mathrm{~mol} / \mathrm{mL}$ at $37^{\circ} \mathrm{C}$ and atmospheric pressure $760 \mathrm{mmHg}$.

2.3. Oxygen-Free Additive. Solutions for taking of additives were prepared by blowing the mixtures with water vapor saturated nitrogen, which was begun an hour before and continued throughout the experiment (Table 1).

Mixtures were heated in thermostat at $37^{\circ} \mathrm{C}$ or cooled by immersing the flask on melting ice. A thickening solution was prepared by dissolving of $\mathrm{KCl}$ in $10 \%$ starch solution up to a concentration $60 \mathrm{~g} / \mathrm{L}$. After mixing $3.5 \mathrm{~mL}$ of the medium with various volumes of thickening additives, the relative viscosity was detected by viscosimeter VK-4 (Russia) in fivefold replicates at $20^{\circ} \mathrm{C}$ (Table 2).

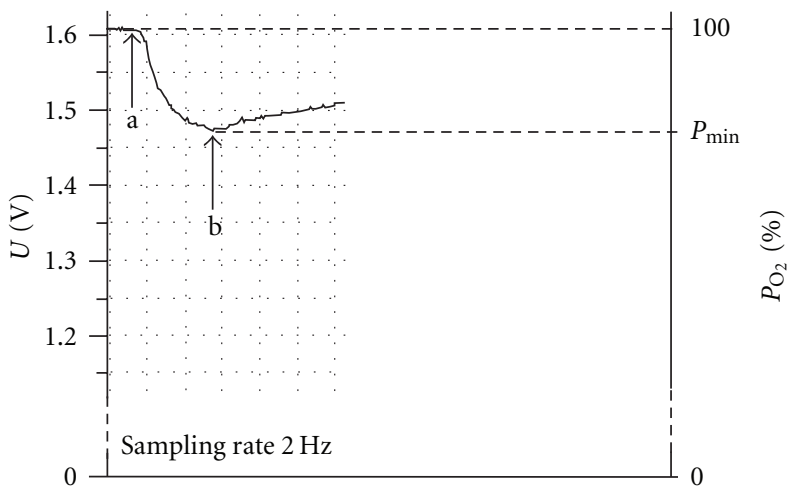

FIGURE 2: Typical registration curve of false oxygen consumption. (a) the time of additive introduction and (b) the minimal value of oxygen partial pressure $\left(P_{\min }\right)$. Recorded by ADC L-154 (LCard, Russia), application PowerGraph (D. Izmailov, Russia). Curve demonstrates a distinct change in the partial pressure of oxygen in the medium in which the proper content of oxygen was not changed. However, the result of such measurement seems to be oxygen consumption and can be interpreted as a consumption of oxygen by the investigated objects under the influence of additives ("false consumption").

TABle 2: Dependence of viscosity on the volume of thickening additive at $20^{\circ} \mathrm{C}$ (mean and coefficient of variation for $n=5$ ).

\begin{tabular}{lcc}
\hline$V$ additive $(\mu \mathrm{L})$ & Relative viscosity of mixture & $C_{V}(\%)$ \\
\hline 0 & 1.06 & 5.2 \\
100 & 1.16 & 4.7 \\
200 & 1.28 & 3.5 \\
300 & 1.36 & 4.0 \\
400 & 1.50 & 0.0 \\
\hline
\end{tabular}

2.4. Scheme of the Experiment. Once incubation medium was oxygen-saturated at $37^{\circ} \mathrm{C}$, then $3.5 \mathrm{~mL}$ of medium was placed in a thermostated measuring cell and covered with a floating lid (Figure 1). This was left aside for 5-15 minutes to stabilize oximeter readings. The recording was turned on. Then, anoxic additive was introduced into the medium by a micropipette through a notch in the lid. Registration curve is shown in Figure 2.

The value of the false oxygen consumption effect $\Delta N$ was determined by the minimum $P_{\min }$ of registration curve (Figure 2)

$$
\Delta N=\frac{5.373 \times 10^{-7} \cdot P_{A} \cdot\left(100-P_{\min }\right)}{760 \cdot 100},
$$




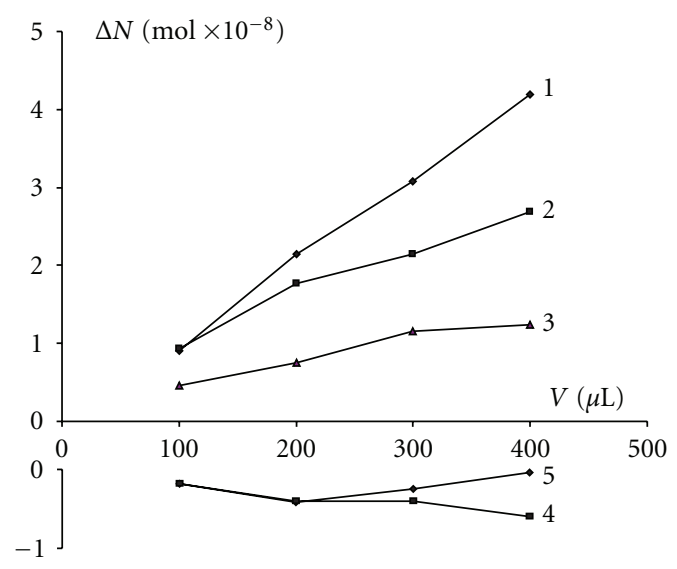

Figure 3: The dependence of false oxygen consumption caused by various factors on the volume of additives. (1) oxygen-free additive, (2) cooled additive, (3) absence of salts in the additive, (4) high concentration of salt in the addition, and 5: high viscosity of additive.

where $5.373 \times 10^{-7}(\mathrm{~mol})$ : the content of $\mathrm{O}_{2}$ in $3.5 \mathrm{~mL}$ of medium $\left(37^{\circ} \mathrm{C}, 760 \mathrm{mmHg}\right)$,

100 (\%): initial reading of oximeter, $P_{\min }(\%)$ : oximeter reading at the minimum, $760(\mathrm{mmHg})$ : standard atmospheric pressure, and $P_{A}(\mathrm{mmHg})$ : atmospheric pressure at the time of measurement.

After each measurement, the cell was filled with a fresh portion of solution saturated with atmospheric oxygen at $37^{\circ} \mathrm{C}$ and kept for 20 minutes for the restoration of initial readings. Additives of 100, 200, 300, and $400 \mu \mathrm{L}$ were used in all cases. Accordingly, 10 readings were obtained for each volume.

In order to determine the spread of viscosity, the coefficient of variation $C_{V}$ was calculated. Furthermore, for the statistical evaluation of study results, the average mean and standard error were also calculated. To demonstrate the statistical significance of false oxygen consumption, $t$-test was used. This test statistically compared the results obtained by the introduction of additives no. 2-5 with the records of additive no. 1 (volume to volume).

\section{Results}

Table 3 represents the mean value of false oxygen consumption $\Delta N$, at the introduction of oxygen free additives. A distinct effect was observed in all cases even for additive of $100 \mu \mathrm{L}$, which is less than $3 \%$ of the incubation medium volume.

Maximum effect was achieved with the introduction of oxygen-free additive cooled to $0^{\circ} \mathrm{C}$ (additive no. 2) and the minimum - by using of additive containing $120 \mathrm{~g} / \mathrm{L} \mathrm{KCl}$ (additive no. 4).

In all experiments, the effect develops in the absence of oxygen in the additives. Therefore, individual contributions of other factors were calculated as differences between the effect developed under simultaneous action of two factors (for additives no. 2-5) and the effect of the oxygen-free additive, which did not differ from the medium by other parameters (additive no. 1). Results are presented in Table 4.

As the table shows, absence of oxygen (additive no. 1), low temperature of additive (additive no. 2), and an absence of the dissolved compounds salting out the oxygen (additive no. 3) caused a statistically significant, but nevertheless a false reduction of oxygen content in the incubation medium. This happened even when the volume of the additive was $100 \mu \mathrm{L}$. Excess of salts in the additive (additive no. 4) caused reliable, but an opposite effect. A more complicated picture was created when thickening additives were used (additive no. $5)$. Therefore, the effect of the viscous additives on the value of false oxygen consumption is presented in graphic form (Figure 3). For comparison, the influence of other factors is shown also.

According to the published data [2], an increase in the viscosity should lead to reduction of the measuring equipment readings and thereby cause the effect of false consumption. However, introduction of viscous additive led to opposite effect (Figure 3). Moreover, statistically confirmed minimum was noted in the curve.

\section{Discussion}

In the present study, by appropriate methods using generally accepted schemes of statistical analysis for biological experiment it was demonstrated that introducing additives, which neither consumed oxygen nor initiated its consumption by other components of the medium, may cause a reproducible and statistically significant decrease in partial pressure of oxygen. Although the content of oxygen in the medium does not change, a decline of partial pressure does not eliminate a possibility of ambiguous interpretation of obtained results $[4,9-11]$.

False oxygen consumption turned out to be not only reproducible, but also quite significant. For example, under combined effects of the factors, even if the additive does not exceed $3 \%(100 \mu \mathrm{L})$ of incubation medium volume, there can be statistically significant "consumption" of more than $4 \%$ of the initial amount of oxygen. With the increase in volume of additive to $11 \%(400 \mu \mathrm{L})$, the false consumption exceeds $15 \%$. This is quite comparable with the real oxygen consumption by objects such as mitochondria in biological experiments $[9,11,12]$.

There are different reasons for the fall of oxygen partial pressure depending on the types of additives. Absence of oxygen in additive (additive no. 1) leads to a dilution of oxygenated medium with pure solvent $[1,4]$. As a result the concentration, but not quantity, of oxygen decreases, leading to a drop in partial pressure. If the additive has a lower temperature than the medium (addition no. 2), its introduction leads to temporary cooling of the mixture. Hence, the solubility of oxygen in the medium increases [5], and the partial pressure decreases. Absence or lower content of oxygen salting out components in the additive (additive no. 3 ) causes a reduction in concentrations of these components in the incubation medium. It also leads to an 
TABLE 3: False oxygen consumption $\Delta N\left(\mathrm{~mol} \times 10^{-8}\right)$, caused by the introduction in the incubation medium of various oxygen-free additives (mean \pm SE, $n=10)$.

\begin{tabular}{|c|c|c|c|c|c|}
\hline \multirow{2}{*}{$V$ additive $(\mu \mathrm{L})$} & \multicolumn{5}{|c|}{ Type of additive (Table 1) } \\
\hline & no. 1 & no. 2 & no. 3 & no. 4 & no. 5 \\
\hline 100 & $0.91 \pm 0.05$ & $1.84 \pm 0.05$ & $1.37 \pm 0.03$ & $0.72 \pm 0.03$ & $0.72 \pm 0.09$ \\
\hline 200 & $2.15 \pm 0.03$ & $3.92 \pm 0.03$ & $2.91 \pm 0.04$ & $1.74 \pm 0.07$ & $1.73 \pm 0.06$ \\
\hline 300 & $3.08 \pm 0.05$ & $5.23 \pm 0.07$ & $4.23 \pm 0.04$ & $2.68 \pm 0.03$ & $2.83 \pm 0.08$ \\
\hline 400 & $4.20 \pm 0.06$ & $6.89 \pm 0.06$ & $5.44 \pm 0.01$ & $3.59 \pm 0.03$ & $4.16 \pm 0.13$ \\
\hline
\end{tabular}

TABLE 4: Individual contributions of separate factors to the total value of false oxygen consumption $\Delta N\left(\mathrm{~mol} \times 10^{-8}\right), n=10$.

\begin{tabular}{lcccc}
\hline$V$ additive $(\mu \mathrm{L})$ & Oxygen concentration & Temperature & $\begin{array}{c}\text { Acting factor } \\
\text { Salting out (no. 3) }\end{array}$ & Salting out (no. 4) \\
\hline 100 & $0.91 \pm 0.05$ & $0.93^{* * *}$ & $0.46^{* * *}$ & $-0.19^{* *}$ \\
200 & $2.15 \pm 0.03$ & $1.77^{* * *}$ & $0.76^{* * *}$ & $-0.41^{* * *}$ \\
300 & $3.08 \pm 0.05$ & $2.15^{* * *}$ & $1.15^{* * *}$ & $-0.19^{* *}$ \\
400 & $4.20 \pm 0.06$ & $2.69^{* * *}$ & $1.24^{* * *}$ & $-0.42^{* * *}$ \\
\hline
\end{tabular}

${ }^{*} P \leq .05 ;{ }^{* *} P \leq .01 ; * * * P \leq .001$. Significant difference in comparison with oxygen-free additive of the same volume.

increase in oxygen solubility $[13,14]$ and a decrease in its partial pressure.

Thus, the additives with high oxygen content, high temperature, or high salt content should cause the opposite effect. However, oversaturated with oxygen and high temperature additives are not commonly used in a biological experiment. The tests were only carried out with additives of salt concentration twofold higher than that of the medium (additive no. 4). The obtained result, as expected, was a statistically significant increase in oxygen partial pressure, which may easily mask the actual oxygen consumption observed in biological study.

The most difficult for interpretation was the experiment with the use of thickening additive (additive no. 5). The increase in viscosity should cause an increase in thickness of electrode diffusion layer [2] and consequently reduce the oxygen sensor readings (the apparent decrease in the partial pressure of oxygen), but the result obtained was the opposite. Moreover, for thickening additives dependence of false consumption effect on the volume of additive has significant minimum, corresponding to volume $200 \mu \mathrm{L}$ (Figure 3). The most probable explanation for this fact may be the simultaneous effect of two factors that have opposite effects on readings of oxygen sensor.

The factor contributing to the reduction of false oxygen consumption effect is the salting-out effect of additive. It contained not only $60 \mathrm{~g} / \mathrm{L} \mathrm{KCl}$ (about 6\%), but also $10 \%$ of starch as a thickener. Their total concentration in the additive was considerably higher than the concentration of solutes in the incubation medium. As a result, the influence of additive no. 5 up to the volume of $200 \mu \mathrm{L}$ was quite similar to the action of additive no. $4(\mathrm{KCl}, 120 \mathrm{~g} / \mathrm{L})$.

The factor contributed to the enhancing of the effect was the increase of viscosity. The diffusion layer of the closed oxygen sensor was large enough, as it included the thickness of the membrane. A slight increase in viscosity of the solution could not have significant influence on its work. The effect began to appear only when the growth of diffusion layer thickness due to the increase in viscosity was comparable to the thickness of the membrane. It is assumed that this occurred when the amount of additive reached $300 \mu \mathrm{L}$, and the relative viscosity of the mixture increased up to 1.36 at $20^{\circ} \mathrm{C}$. Starting from this volume, viscosity determined the character of dependence (Figure 3).

\section{Conclusion}

Thus, we can conclude that introduction of any additive to the incubation medium, which differs from medium in composition, temperature, or viscosity, will unavoidably lead to changes in oxygen sensor readings. These changes can be large enough to cause a significant increase in measurement error, and also an appearance of well-reproducible artifact. This is identified as the false oxygen consumption effect. Under certain conditions, this effect can reach high percentage of the oxygen content in the incubation medium.

Exclusion of such distortions is not possible, because additives will always be different from the incubation medium by various parameters. Nevertheless, it is necessary to minimize the effect. For this purpose, reagent solutions saturated with atmospheric oxygen at the temperature of incubation medium should be used. When the reagents with low concentrations are prepared, water should be substituted by incubation medium. If these recommendations are difficult to fulfill, additives should be introduced with control measurements of oxygen partial pressure changes in the conditions that prevent real consumption of oxygen in the incubation medium.

\section{References}

[1] B. Chance and G. R. Williams, "Respiratory enzymes in oxidative phosphorylation. I. Kinetics of oxygen utilization," 
The Journal of Biological Chemistry, vol. 217, no. 1, pp. 383393, 1955.

[2] A. A. Shpakov and L. F. Panchenko, "Nature of the effect "rapid oxygen consumption" during the introduction of tissue homogenate or a mitochondrial suspension into the polarographic cell," Biofizika, vol. 20, no. 3, pp. 467-472, 1975.

[3] E. N. Kotova and I. S. Rotenberg, "Nature of rapid oxygen consumption by mitochondria following their placement in a polarography," Biofizika, vol. 22, no. 4, pp. 746-747, 1977.

[4] E. Gnaiger, "Bioenergetics at low oxygen: dependence of respiration and phosphorylation on oxygen and adenosine diphosphate supply," Respiration Physiology, vol. 128, no. 3, pp. 277-297, 2001.

[5] A. Barrientos, "In vivo and in organello assessment of OXPHOS activities," Methods, vol. 26, no. 4, pp. 307-316, 2002.

[6] L. C. Clark Jr., R. Wolf, D. Granger, and Z. Taylor, "Continuous recording of blood oxygen tensions by polarography," Journal of Applied Physiology, vol. 6, no. 3, pp. 189-193, 1953.

[7] K. H. Mancy, D. A. Okun, and C. N. Reilley, "A galvanic cell oxygen analyzer," Journal of Electroanalytical Chemistry, vol. 4, no. 2, pp. 65-92, 1962.

[8] M. V. Miniaev and L. I. Voronchikhina, "Ambient air interference in oxygen intake measurements in liquid incubating media with the use of open polarographic cells," Aviakosmicheskaia i Ekologicheskaia Meditsina, vol. 41, no. 2, pp. 64-68, 2007.

[9] A. Barrientos, L. Kenyon, and C. T. Moraes, "Human xenomitochondrial cybrids: cellular models of mitochondrial complex i deficiency," Journal of Biological Chemistry, vol. 273, no. 23, pp. 14210-14217, 1998.

[10] C. Pourplanche, V. Larreta-Garde, and D. Thomas, "Comparison of polarographic and chemical measurements of oxygen uptake in complex media: the example of lipoxygenase reaction," Analytical Biochemistry, vol. 198, no. 1, pp. 160-164, 1991.

[11] E. P. Dassa, E. Dufour, S. Goncalves, H. T. Jacobs, and P. Rustin, "The alternative oxidase, a tool for compensating cytochrome c oxidase deficiency in human cells," Physiologia Plantarum, vol. 137, no. 4, pp. 427-434, 2009.

[12] C. J. Kay and J. M. Palmer, "Solubilization of the alternative oxidase of cuckoo-pint (Arum maculatum) mitochondria. Stimulation by high concentrations of ions and effects of specific inhibitors," The Biochemical Journal, vol. 228, no. 2, pp. 309-318, 1985.

[13] J. Mexal, J. T. Fisher, J. Osteryoung, and C. P. Reid, "Oxygen availability in polyethylene glycol solutions and its implications in plant-water relations," Plant Physiology, vol. 55, no. 1, pp. 20-24, 1975.

[14] A. M. Klibanov, N. O. Kaplan, and M. D. Kamenhy, "A rationale for stabilization of oxygen labile enzymes: application to a clostridial hydrogenase," Proceedings of the National Academy of Sciences of the United States of America, vol. 75, no. 8, pp. 3640-3643, 1978. 


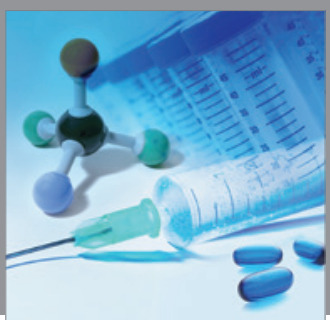

International Journal of

Medicinal Chemistry

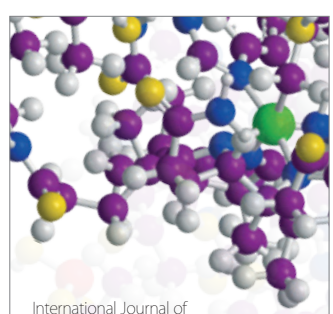

Carbohydrate Chemistry

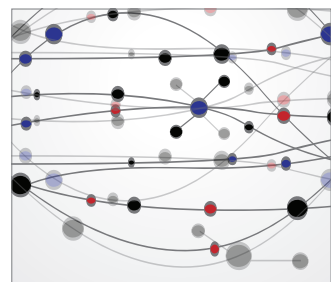

The Scientific World Journal
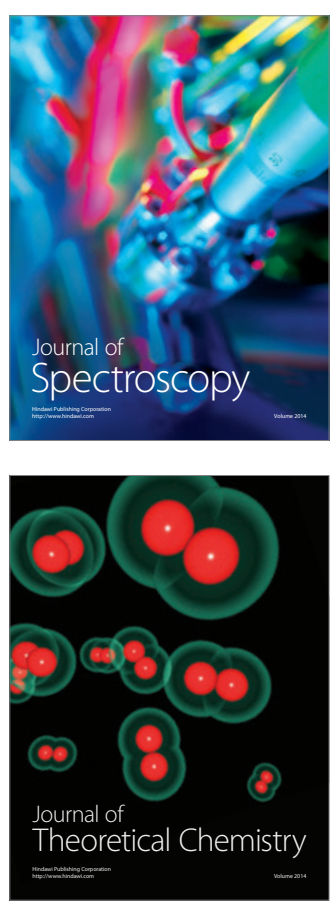
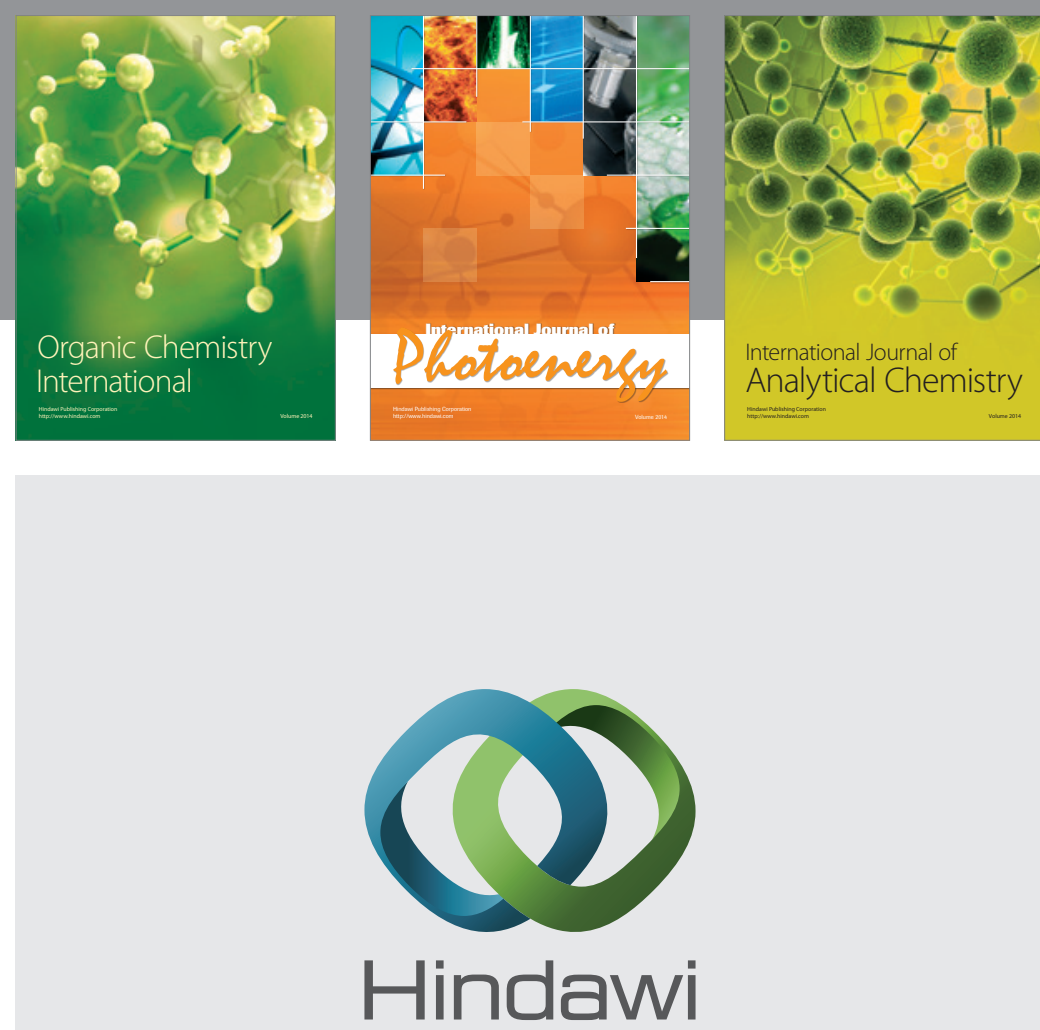

Submit your manuscripts at

http://www.hindawi.com
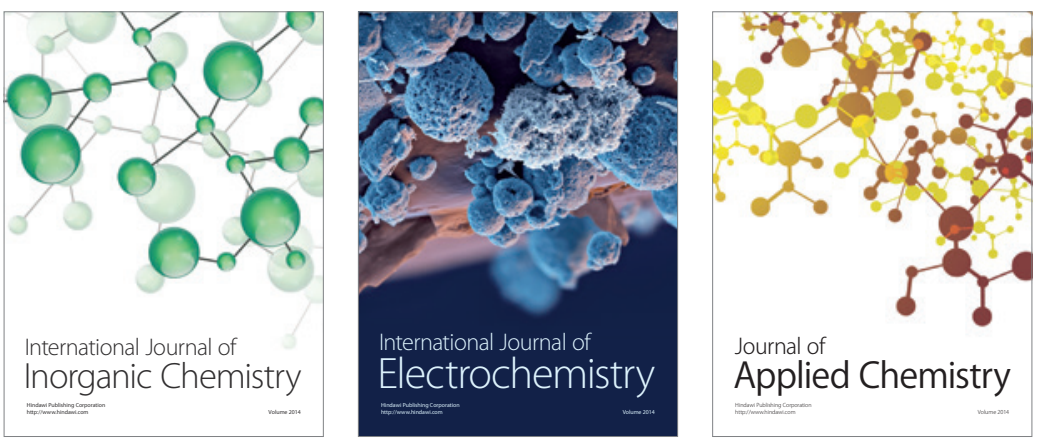

Journal of

Applied Chemistry
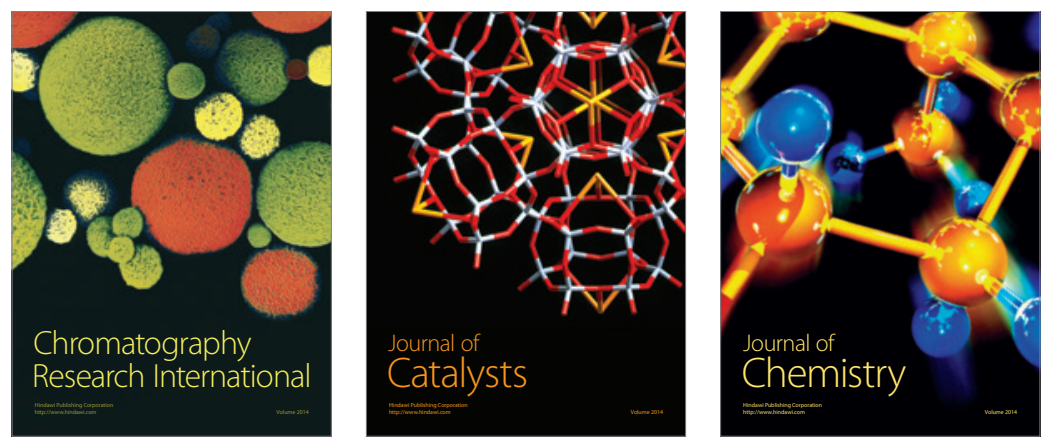
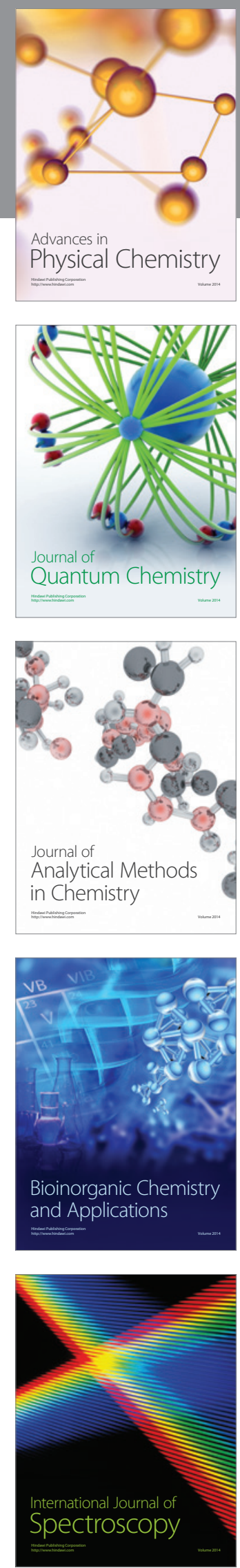